\title{
About the Significance of Quasinormal Modes of Black Holes
}

\author{
Hans-Peter Nollert \\ Theoretische Astrophysik, Computational Physics, Universität Tübingen \\ 72076 Tübingen, Germany
}

\begin{abstract}
Quasinormal modes have played a prominent role in the discussion of perturbations of black holes, and the question arises whether they are as significant as normal modes are for self adjoint systems, such as harmonic oscillators. They can be significant in two ways: Individual modes may dominate the time evolution of some perturbation, and a whole set of them could be used to completely describe this time evolution. It is known that quasinormal modes of black holes have the first property, but not the second. It has recently been suggested that a discontinuity in the underlying system would make the corresponding set of quasinormal modes complete. We therefore turn the Regge-Wheeler potential, which describes perturbations of Schwarzschild black holes, into a series of step potentials, hoping to obtain a set of quasinormal modes which shows both of the above properties. This hope proves to be futile, though: The resulting set of modes appears to be complete, but it does not contain any individual mode any more which is directly obvious in the time evolution of initial data. Even worse: The quasinormal frequencies obtained in this way seem to be extremely sensitive to very small changes in the underlying potential. The question arises whether - and how - it is possible to make any definite statements about the significance of quasinormal modes of black holes at all, and whether it could be possible to obtain a set of quasinormal modes with the desired properties in another way.
\end{abstract}

PACS codes: 97.60.Lf, 04.30.+x, 11.10.Qr, 02.60.+y

Paper: gr-qc/9602032

\section{Introduction}

Quasinormal modes are single frequency modes dominating the time evolution of perturbations of systems which are subject to damping, either by internal dissipation or by radiating away energy. Due to the damping, the frequency of a quasinormal mode must be complex, its imaginary part being inversely proportional to the typical damping time. Examples are oscillations of stars damped by internal friction. In general relativity, damping occurs even without friction, since energy may be radiated away towards infinity by gravitational waves. This leads to a new class of quasinormal modes of neutron stars which are not present in the Newtonian case $[1,2]$. Recently it has also been suggested $[3,4]$ to describe the behavior of leaky optical cavities using a quasinormal mode formalism, rather than studying the surrounding infinite system that the cavity is embedded in. 
Even linearized perturbations of black holes exhibit quasinormal modes [5], despite the absence of any oscillating fluid. The characteristic modes of black holes have first been found by numerical calculations [6], leading to attempts to develop a formalism to describe characteristic oscillations without having to refer to a specific initial perturbation, based only on the properties of the underlying system.

The study of linearized perturbations of Schwarzschild black holes leads to the Schrödinger-like equation (e.g. [7])

$$
\psi^{\prime \prime}(x)+\left(\omega^{2}-V(x)\right) \psi(x)=0,
$$

where $\psi$ represents the wave function of the perturbation and $V(x)$ is the so-called ReggeWheeler potential

$$
V(x)=\left(1-\frac{1}{r}\right)\left(\frac{l(l+1)}{r^{2}}-\frac{3}{r^{3}}\right) .
$$

$l$ originates from the expansion of the perturbation in terms of spherical harmonics, and $r$ is considered a function of $x$ such that $x=r+\ln (r-1)$. All units are chosen such that $G=1$ and $c=1$, the radial coordinate is scaled such that the horizon is at $r=1$, i.e. $2 M=1$, where $M$ is the mass of the black hole.

Quasinormal modes are formally defined by those solutions of Eq. (1) which satisfy purely outgoing boundary conditions, i.e.

$$
\psi(x) \stackrel{x \rightarrow-\infty}{\longrightarrow} e^{+i \omega x} \quad \text { and } \quad \psi(x) \stackrel{x \rightarrow+\infty}{\longrightarrow} e^{-i \omega x} .
$$

We have chosen the time dependence to be $e^{i \omega t}$, quasinormal frequencies of stable systems must therefore have a positive imaginary part.

In the case of a Schwarzschild black hole, it is known [8] that there is an infinite number of discrete frequencies $\omega$ which allow solutions of Eqs. (1) - (3). However, the significance of these frequencies and their associated modes is not immediately clear. In the case of normal modes, the underlying mathematical problem is self adjoint, and the normal modes form a complete set of solutions. Therefore, the behavior of the system can be analyzed completely in terms of its normal modes. There is no such theorem for quasinormal modes, since Eqs. (1) - (3) do not define a self adjoint problem. The completeness of a set of normal modes also makes it possible to define unambiguously what we mean by the excitation strength of a given normal mode as a result of some initial data. Again, this definition does not simply carry over to quasinormal modes, not even to the fundamental ones which are obvious in the time evolved data.

Nevertheless, quasinormal modes are believed to play a significant role for the study of perturbations of black holes. This view is supported by the fact that the fundamental quasinormal frequency (the one with the smallest imaginary part) is generally found to dominate the time evolution of some initial perturbation. Even a few of the more strongly damped modes, which are not immediately obvious in the time evolved data, can be extracted with suitable techniques [9]. 
However, the fundamental quasinormal mode can only be an approximation of the actual time evolved perturbation. An interesting question is whether the set of all quasinormal modes of a black hole can be used, in analogy to a set of normal modes of a self adjoint system, to completely describe the behavior of a perturbation of the black hole, i.e. the time evolution of some initial data for the perturbation equation. Quasinormal modes cannot be complete in the usual sense, meaning that at any given time, the solution can be represented, over all space, as a sum of quasinormal modes. Due to the boundary conditions (3), solutions corresponding to damped oscillations have to grow exponentially as $x \rightarrow \pm \infty$.

However, it may be possible to represent the time dependence of a solution at a given point in space completely as a sum over quasinormal modes. A model systems with this property has been studied by Price and Husain [10]. In general, however, quasinormal modes do not form such a complete system. This is related to Eq. (1), together with the boundary conditions (3), not defining a selfadjoint problem. Therefore, the question of the mathematical as well as physical meaning of quasinormal modes and of their usefulness in practical computations has to be studied for every system in particular.

Ching et al. [11] have argued that a system which is described by a Schrödinger-like equation such as Eq. (1) will indeed have a complete set of quasinormal modes if the potential $V(x)$ has a discontinuity either in the potential itself or in any of its derivatives.

On the other hand, it is known that the quasinormal modes of a Schwarzschild black hole (or of a neutron star) cannot be complete. One reason is that the late time behavior of perturbations of a black hole is first dominated by damped oscillations ("quasinormal ringing"), but at very late times it exhibits a power-law tail [9]. Such a tail cannot be obtained by a sum of quasinormal modes. In addition, the set of quasinormal frequencies of black holes does not contain frequencies with large real parts, i.e. oscillations with very short periods. Therefore, any initial data corresponding to such oscillations cannot be adequately represented by quasinormal modes.

The argument of Ching et al. [11] therefore implies that if the (smooth) Regge-Wheeler potential is changed slightly such that a discontinuity, however small, is introduced in the potential or any of its derivatives, the set of quasinormal modes of the black hole will change from incomplete to complete. On the one hand, this would be very convenient, since it would allow the study of arbitrary perturbations in terms of this expanded set of quasinormal modes, just as the time dependent behavior of any self adjoint system can be studied in terms of its normal modes. On the other hand, one would not expect the physical response of the black hole, i.e. the time evolution of some perturbation, to be significantly affected by a small change in the potential. We are therefore facing a possible contradiction: The actual time evolution of some initial data will probably not be affected significantly by this small change in the potential, while the quasinormal mode spectrum, which is generally believed to represent crucial aspects of this time evolution, should change drastically.

In an attempt to shed some light on this apparent contradiction, we will replace the Regge-Wheeler potential in Eq. (1) by a step potential. This is a potential which is 
piecewise constant, constructed in such a way that it approximates the original, continuous Regge-Wheeler potential. Of course, we expect any given step potential to have a quasinormal modes spectrum which is somewhat different from that of the smooth Regge-Wheeler potential. Therefore, we construct a whole series of such step potentials and study the behavior of their quasinormal frequencies as the number of steps is increased, i.e. as the step potentials are allowed to approximate the continuous Regge-Wheeler potential better and better.

\section{Procedure}

In the following, we will always use the Regge-Wheeler potential (2) with $l=2$. There are, of course, many different ways to construct step potentials which eventually approximate a given smooth potential. We will present the results for three such possibilities:

(i) The difference in potential between steps is (roughly) constant. In order to achieve this, the length of the steps is variable and depends essentially on the derivative of the smooth potential.

(ii) The length of the steps is constant, while the difference in height between the steps depends on the smooth potential. However, the length of the steps may be chosen differently to the left and to the right of the maximum of the potential if the potential is not symmetric around its maximum.

(iii) As in (i), but an exponential damping is applied to the smooth potential before the steps are constructed.

In all cases, the value of the step potential for a particular step is generally determined by

$$
V_{\mathrm{Step}}\left(x_{n-1} \leq x<x_{n}\right)=\frac{1}{x_{n}-x_{n-1}} \int_{x_{n-1}}^{x_{n}} V(x) d x \quad \text { for } \quad n=-N_{\mathrm{St}}+1 \ldots N_{\mathrm{St}}
$$

where $N_{\mathrm{St}}$ is the number of steps to either the left or the right of the maximum of the

potential. For method (i), the position $x_{N_{\mathrm{St}}}$ of the last jump on the right is determined by

$$
V_{\mathrm{Step}}\left(x_{N_{\mathrm{St}}-1} \leq x<x_{N_{\mathrm{St}}}\right)=\frac{V_{\mathrm{max}}}{2 N_{\mathrm{St}}}=\frac{1}{x_{N_{\mathrm{St}}}-x_{N_{\mathrm{St}}-1}} \int_{x_{N_{\mathrm{St}}-1}}^{\infty} V(x) d x,
$$

with a corresponding condition for the last step on the left. This ensures that

$$
\int_{-\infty}^{+\infty} V_{\text {Step }}(x) d x=\int_{-\infty}^{+\infty} V(x) d x .
$$

This condition is not satisfied by methods (ii) and (iii).

Figure 1 (i) - (iii) shows the resulting step potentials for different numbers of steps. Method (i) obviously leads to the last step on the right of the potential's maximum becoming extremely long. This is due to the slow decay of the Regge-Wheeler potential for 

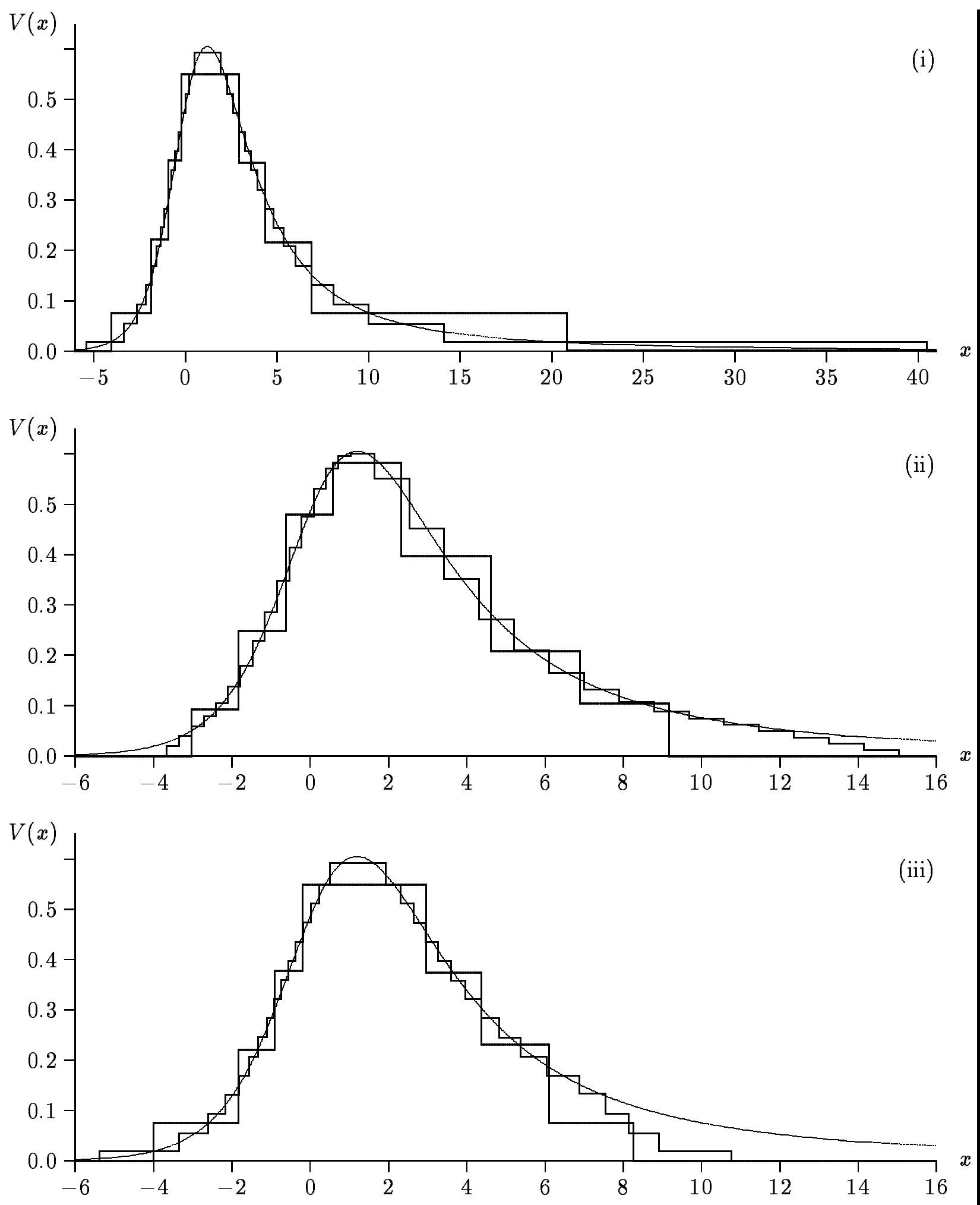

Figure 1: Step potentials constructed from the Regge-Wheeler potential, with $N_{\mathrm{St}}=4$ and $N_{\mathrm{St}}=16$ and construction principles (i) - (iii). The smooth Regge-Wheeler potential is also shown for comparison. 
$x \rightarrow \infty: V(x) \sim 1 / x^{2}$ as $x \rightarrow \infty$, while $V(x) \sim \exp (x)$ as $x \rightarrow-\infty$, as can be verified from Eq. (2). The last step being so much longer compared to all other steps seems rather artificial. In fact, with increasing $N_{\text {St }}$, the last steps grows longer, while the steps near the maximum of the potential become shorter, as one would indeed expect for a refinement of the step potential.

Therefore, in method (iii) we have imposed an exponential damping on the potential before constructing the steps:

$$
\tilde{V}(x)= \begin{cases}V(x) & x<=x_{\text {cut }} \\ V(x) / \cosh ^{2}\left(\frac{1}{2}\left(x-x_{\text {cut }}\right)\right) & x>x_{\text {cut }}\end{cases}
$$

Note that this leads to an exponential decrease with the same power as for $x \rightarrow-\infty$. Of course, $x_{\text {cut }}$ itself has to grow with increasing $N_{\mathrm{St}}$ if the step potential is supposed to approximate the smooth potential arbitrarily well for $N_{\mathrm{St}} \rightarrow \infty$. We use $x_{\text {cut }}:=x_{\max }+$ $4\left(N_{\mathrm{St}} / 4\right)^{1 / 4}$, where the maximum of the Regge-Wheeler potential is at $x_{\max } \simeq 1.1947$.

A related problem occurs in method (ii): Here, the last step would become much higher than the differences in height of the preceding steps, again due to the slow decrease of the Regge-Wheeler potential for $x \rightarrow \infty$. In order to avoid this, several steps before the last are reduced in height such that the step height approaches 0 linearly. Again, this artificial decrease sets in at larger values of $x$ if the number of steps increases.

The determination of the quasinormal modes of these step potentials is quite simple: On the $n$th step, the solution is given by $\psi\left(x_{n-1} \leq x<x_{n}\right)=A_{n} e^{i k_{n} x}+B_{n} e^{-i k_{n} x}$, with $k_{n}=\sqrt{\omega^{2}-V_{\text {Step }}(x)}$, and $A_{n}, B_{n}$ determined according to the boundary conditions. For a given (complex) frequency $\omega$, we start with $\psi_{+\infty}=e^{-i \omega x}$ to the right of the potential and, moving to the left, determine $A_{n}$ and $B_{n}$ at every step by the standard procedure of matching $\psi(x)$ and $\psi^{\prime}(x)$ at every step. If $\psi(x)=\psi_{-\infty}(x)=e^{+i \omega x}$ after reaching the left side of the support of the potential, i.e. if $B_{-N_{\mathrm{St}}}=0$, the frequency $\omega$ is a quasinormal frequency.

\section{Results}

The quasinormal frequencies determined in this way are shown in Fig. 2. The details of the behavior of the step potentials' quasinormal frequencies, such as the value of the fundamental frequency, the imaginary parts of the 'lined up' frequencies, and even their spacing, depend on the method of construction. The dominating features, however, are independent of it. In all cases, the spacing between the frequencies becomes closer as the number of steps increases, but they generally occupy the same part of the complex plane. In particular, they remain 'lined up' more or less parallel to the real axis, rather than to the imaginary axis as the quasinormal frequencies of the smooth Regge-Wheeler potential. There is no indication that the frequencies cease to reach arbitrarily large real parts, i.e. arbitrarily small oscillation periods, as the number of steps becomes very large.

As $N_{\text {St }}$ increases, there are individual frequencies 'escaping' from the line of frequencies towards the imaginary axis. However, even these frequencies do not seem to approach the quasinormal frequencies of the Regge-Wheeler potential. 

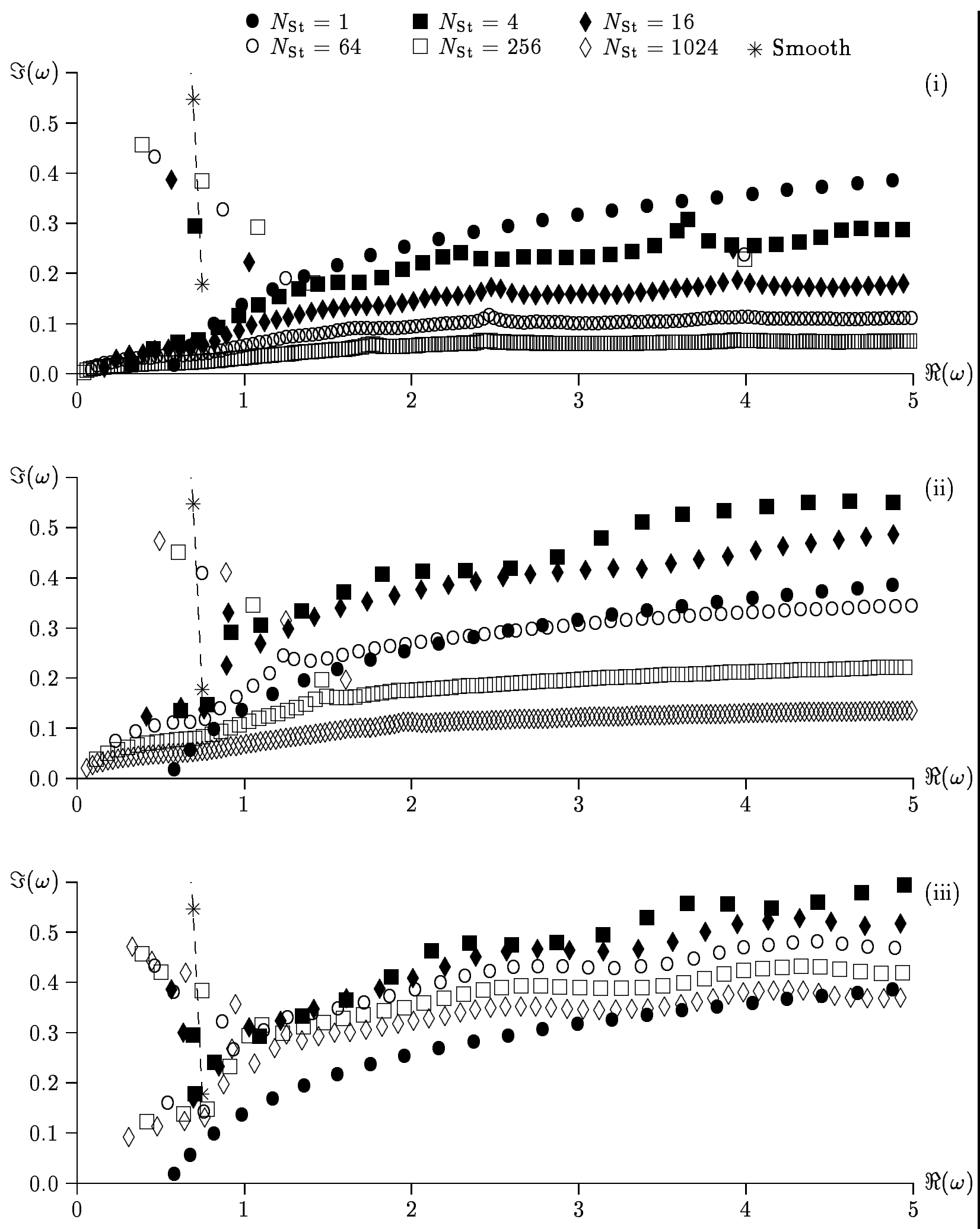

Figure 2: Quasinormal frequencies for step potentials constructed using methods (i) - (iii). The quasinormal frequencies of the smooth Regge-Wheeler potential are included for comparison. 


\begin{tabular}{|c|c|c|c|c|c|c|c|c|c|c|}
\hline & \multicolumn{3}{|c|}{ (i) Variable step size } & \multicolumn{3}{|c|}{ (ii) Fixed step size } & \multicolumn{3}{|c|}{ (iii) Exponential cutoff } & smooth \\
\hline Index & 4 steps & 64 & 256 & 4 steps & 64 & 1024 & 4 steps & 64 & 1024 & \\
\hline 1 & $\begin{array}{c}0.329 \\
0.021\end{array}$ & $\begin{array}{c}0.084 \\
0.006\end{array}$ & $\begin{array}{c}0.042 \\
0.003\end{array}$ & $\begin{array}{c}0.621 \\
0.136\end{array}$ & $\begin{array}{c}0.229 \\
0.073\end{array}$ & $\begin{array}{c}0.060 \\
0.020\end{array}$ & $\begin{array}{c}0.696 \\
0.180\end{array}$ & $\begin{array}{c}0.756 \\
0.140\end{array}$ & $\begin{array}{c}0.311 \\
0.091\end{array}$ & \\
\hline 2 & $\begin{array}{c}0.457 \\
0.051\end{array}$ & $\begin{array}{c}0.120 \\
0.015\end{array}$ & $\begin{array}{c}0.061 \\
0.008\end{array}$ & $\begin{array}{r}0.782 \\
0.147\end{array}$ & $\begin{array}{c}0.352 \\
0.093\end{array}$ & $\begin{array}{c}0.093 \\
0.026\end{array}$ & $\begin{array}{c}0.695 \\
0.292\end{array}$ & $\begin{array}{c}0.533 \\
0.157\end{array}$ & $\begin{array}{c}0.485 \\
0.113\end{array}$ & \\
\hline 3 & $\begin{array}{c}0.604 \\
0.064\end{array}$ & $\begin{array}{c}0.162 \\
0.020\end{array}$ & $\begin{array}{c}0.082 \\
0.010\end{array}$ & $\begin{array}{c}0.922 \\
0.292\end{array}$ & $\begin{array}{c}0.464 \\
0.104\end{array}$ & \begin{tabular}{|c|}
0.124 \\
0.030
\end{tabular} & $\begin{array}{c}0.816 \\
0.232\end{array}$ & $\begin{array}{c}0.924 \\
0.259\end{array}$ & \begin{tabular}{|c|}
0.643 \\
0.123
\end{tabular} & \\
\hline 4 & $\begin{array}{c}0.727 \\
0.068\end{array}$ & $\begin{array}{c}0.202 \\
0.022\end{array}$ & \begin{tabular}{|c|}
0.102 \\
0.011
\end{tabular} & $\begin{array}{c}1.101 \\
0.306\end{array}$ & $\begin{array}{c}0.574 \\
0.110\end{array}$ & \begin{tabular}{|c|}
0.156 \\
0.033
\end{tabular} & $\begin{array}{c}1.079 \\
0.287\end{array}$ & $\begin{array}{c}1.111 \\
0.298\end{array}$ & \begin{tabular}{|c|}
0.766 \\
0.131
\end{tabular} & \\
\hline 5 & $\begin{array}{r}0.847 \\
0.093\end{array}$ & $\begin{array}{c}0.241 \\
0.025\end{array}$ & \begin{tabular}{|c|}
0.122 \\
0.013
\end{tabular} & $\begin{array}{c}.346 \\
0.335\end{array}$ & \begin{tabular}{|c|}
0.677 \\
0.112
\end{tabular} & \begin{tabular}{|c|}
0.187 \\
0.035
\end{tabular} & $\begin{array}{c}1.335 \\
0.326\end{array}$ & $\begin{array}{c}1.253 \\
0.325\end{array}$ & \begin{tabular}{|c|}
0.879 \\
0.197
\end{tabular} & \\
\hline 10 & $\begin{array}{c}1.443 \\
0.180\end{array}$ & $\begin{array}{c}0.441 \\
0.031\end{array}$ & $\begin{array}{r}0.225 \\
0.016\end{array}$ & $\begin{array}{c}2.594 \\
0.419\end{array}$ & \begin{tabular}{|c|}
1.154 \\
0.208
\end{tabular} & \begin{tabular}{|c|}
0.338 \\
0.043
\end{tabular} & $\begin{array}{c}2.569 \\
0.468\end{array}$ & $\begin{array}{c}2.007 \\
0.380\end{array}$ & $\begin{array}{c}1.351 \\
0.284\end{array}$ & \\
\hline 20 & $\begin{array}{c}2.673 \\
0.234\end{array}$ & $\begin{array}{c}0.824 \\
0.043\end{array}$ & \begin{tabular}{|c|}
0.427 \\
0.021
\end{tabular} & $\begin{array}{r}5.150 \\
0.550\end{array}$ & $\begin{array}{c}2.060 \\
0.271\end{array}$ & $\begin{array}{c}0.635 \\
0.051\end{array}$ & $\begin{array}{c}5.133 \\
0.599\end{array}$ & $\begin{array}{c}3.502 \\
0.431\end{array}$ & $\begin{array}{c}2.296 \\
0.340\end{array}$ & \\
\hline 50 & $\begin{array}{c}6.460 \\
0.323\end{array}$ & $\begin{array}{c}1.957 \\
0.091\end{array}$ & $\begin{array}{c}1.023 \\
0.033\end{array}$ & $\begin{array}{r}12.862 \\
0.710\end{array}$ & \begin{tabular}{|c|}
4.897 \\
0.342
\end{tabular} & \begin{tabular}{|c|}
1.517 \\
0.093
\end{tabular} & $\begin{array}{r}12.808 \\
0.737\end{array}$ & $\begin{array}{c}8.032 \\
0.527\end{array}$ & $\begin{array}{c}5.125 \\
0.377\end{array}$ & \\
\hline 100 & \begin{tabular}{|r|}
12.758 \\
0.365
\end{tabular} & $\begin{array}{c}3.788 \\
0.110\end{array}$ & \begin{tabular}{|c|}
2.027 \\
0.057
\end{tabular} & \begin{tabular}{|}
25.720 \\
0.829
\end{tabular} & $\begin{array}{c}9.684 \\
0.392\end{array}$ & $\begin{array}{c}2.874 \\
0.120\end{array}$ & $\begin{array}{r}25.623 \\
0.855\end{array}$ & \begin{tabular}{|r|}
15.602 \\
0.583
\end{tabular} & $\begin{array}{c}9.837 \\
0.424\end{array}$ & \\
\hline o1 & $\begin{array}{c}0.707 \\
0.295\end{array}$ & $\begin{array}{c}1.250 \\
0.189\end{array}$ & $\begin{array}{c}1.415 \\
0.182\end{array}$ & & $\begin{array}{r}0.749 \\
0.408\end{array}$ & $\begin{array}{c}1.610 \\
0.197\end{array}$ & & $\begin{array}{c}0.872 \\
0.320\end{array}$ & $\begin{array}{c}0.951 \\
0.355\end{array}$ & $\begin{array}{r}0.747 \\
0.178\end{array}$ \\
\hline$o 2$ & & $\begin{array}{r}0.875 \\
0.327\end{array}$ & $\begin{array}{r}1.084 \\
0.294\end{array}$ & & & $\begin{array}{r}1.254 \\
0.315\end{array}$ & & $\begin{array}{r}0.578 \\
0.376\end{array}$ & $\begin{array}{r}0.652 \\
0.419\end{array}$ & $\begin{array}{r}0.693 \\
0.548\end{array}$ \\
\hline o3 & & $\begin{array}{c}0.465 \\
0.432\end{array}$ & $\begin{array}{r}0.751 \\
0.385\end{array}$ & & & \begin{tabular}{|c|}
0.893 \\
0.411
\end{tabular} & & $\begin{array}{c}0.465 \\
0.432\end{array}$ & $\begin{array}{c}0.450 \\
0.442\end{array}$ & $\begin{array}{r}0.602 \\
0.957\end{array}$ \\
\hline o4 & & $\begin{array}{c}3.991 \\
0.236\end{array}$ & \begin{tabular}{|c|}
0.391 \\
0.457
\end{tabular} & & & \begin{tabular}{|c|}
0.496 \\
0.473
\end{tabular} & & & $\begin{array}{l}0.335 \\
0.471\end{array}$ & \\
\hline 05 & & & \begin{tabular}{|c|}
3.997 \\
0.229
\end{tabular} & & & & & & & \\
\hline
\end{tabular}

Table 1: Quasinormal frequencies of step potentials for all three methods of construction and various step sizes. Each entry lists the real part of the frequency as the upper left and the imaginary part as the lower right number. The first three frequencies of the smooth Regge-Wheeler potential are included for comparison.

Table 1 lists some quasinormal frequencies for all three methods of construction and different numbers of steps, along with the 'outlying' frequencies and the fundamental quasinormal frequencies of the smooth Regge-Wheeler potential. In the case of method (i), the long step at large $x$ causes numerical instabilities. Therefore, results for $N_{\mathrm{St}}=1024$ are not available; the frequencies for $N_{\mathrm{St}}=256$ are listed instead.

In order to check whether the behavior we found could be the result of some numerical artifact, we have explicitly calculated the time evolution of some Cauchy data subject to 
the time dependent version of Eq. (1)

$$
\frac{\partial^{2} \psi}{\partial x^{2}}-\frac{\partial^{2} \psi}{\partial t^{2}}-V(x) \psi=0,
$$

inserting step potentials constructed using method (i), with $N_{\mathrm{St}}=1, N_{\mathrm{St}}=4$, and $N_{\mathrm{St}}=$ 64, for $V(x)$. The initial data consisted of a bell shaped peak with compact support, incident onto the potential from the left, the point of observation is at $x=120$. The result for $N_{\mathrm{St}}=4$ is shown in Fig. 3 , and it manages to combine two seemingly contradictory requirements: It is only slightly different from the time evolution data for the continuous Regge-Wheeler potential (included in Fig. 3 for comparison), and it also represents the totally different spectrum of quasinormal frequencies of the step potential. The similarity is seen shortly after the initial burst has passed (Fig. 3(i)); actually, the time evolution here is dominated by the fundamental quasinormal frequency of the smooth potential, which is not present in the spectrum of the step potential. At very late times, however, the perturbation oscillates with the fundamental frequency of the step potential (Fig. 3(ii)), while the data for the smooth potential is damped more strongly and is therefore invisible in Fig. 3(ii). However, this difference concerns only a tiny fraction of the total energy radiated by the system.

\begin{tabular}{|c|cc|cc|cc|cc|}
\hline & \multicolumn{3}{|c|}{ Stationary calculation } & \multicolumn{3}{c|}{ Time evolution } \\
\hline & \multicolumn{2}{|c|}{1 step } & \multicolumn{2}{|c|}{64 steps } & \multicolumn{2}{c|}{ 1 step } & \multicolumn{2}{c|}{ 64 steps } \\
\hline Index & $\operatorname{Re}(\omega)$ & $\operatorname{Im}(\omega)$ & $\operatorname{Re}(\omega)$ & $\operatorname{Im}(\omega)$ & $\operatorname{Re}(\omega)$ & $\operatorname{Im}(\omega)$ & $\operatorname{Re}(\omega)$ & $\operatorname{Im}(\omega)$ \\
\hline 1 & 0.5836 & 0.0165 & 0.0841 & 0.0064 & 0.5828 & 0.0169 & 0.0841 & 0.0064 \\
2 & 0.6786 & 0.0550 & 0.1203 & 0.0151 & 0.6792 & 0.0566 & 0.1203 & 0.0152 \\
3 & 0.8187 & 0.0975 & & & 0.8245 & 0.1022 & & \\
4 & 0.9859 & 0.1351 & & & 1.0333 & 0.1374 & & \\
\hline
\end{tabular}

Table 2: Comparison of quasinormal frequencies obtained from the stationary calculation described in this paper with frequencies obtained by a fit of time evolution data.

We determined the dominating frequencies of these late time oscillations by fitting quasinormal modes to the time dependent solution, their frequencies not being fixed, but parameters of the fit procedure. The fundamental frequencies obtained in this way are given in Tab. 2, they agree very well with the fundamental quasinormal frequencies obtained from the stationary calculation. Some deviation is expected, since the time dependent calculation works on a fixed grid and does not know about the exact location of the jumps in the step potential, or even about the potential being a step potential at all.

\section{Discussion}

Introducing discontinuities in the Regge-Wheeler potential has a significant influence on the quasinormal mode spectrum, even if the jumps become very small. Most of the frequencies become 'lined up' roughly parallel to the real axis, as they do for the simple square barrier potential, rather than being lined up along the imaginary axis like the 

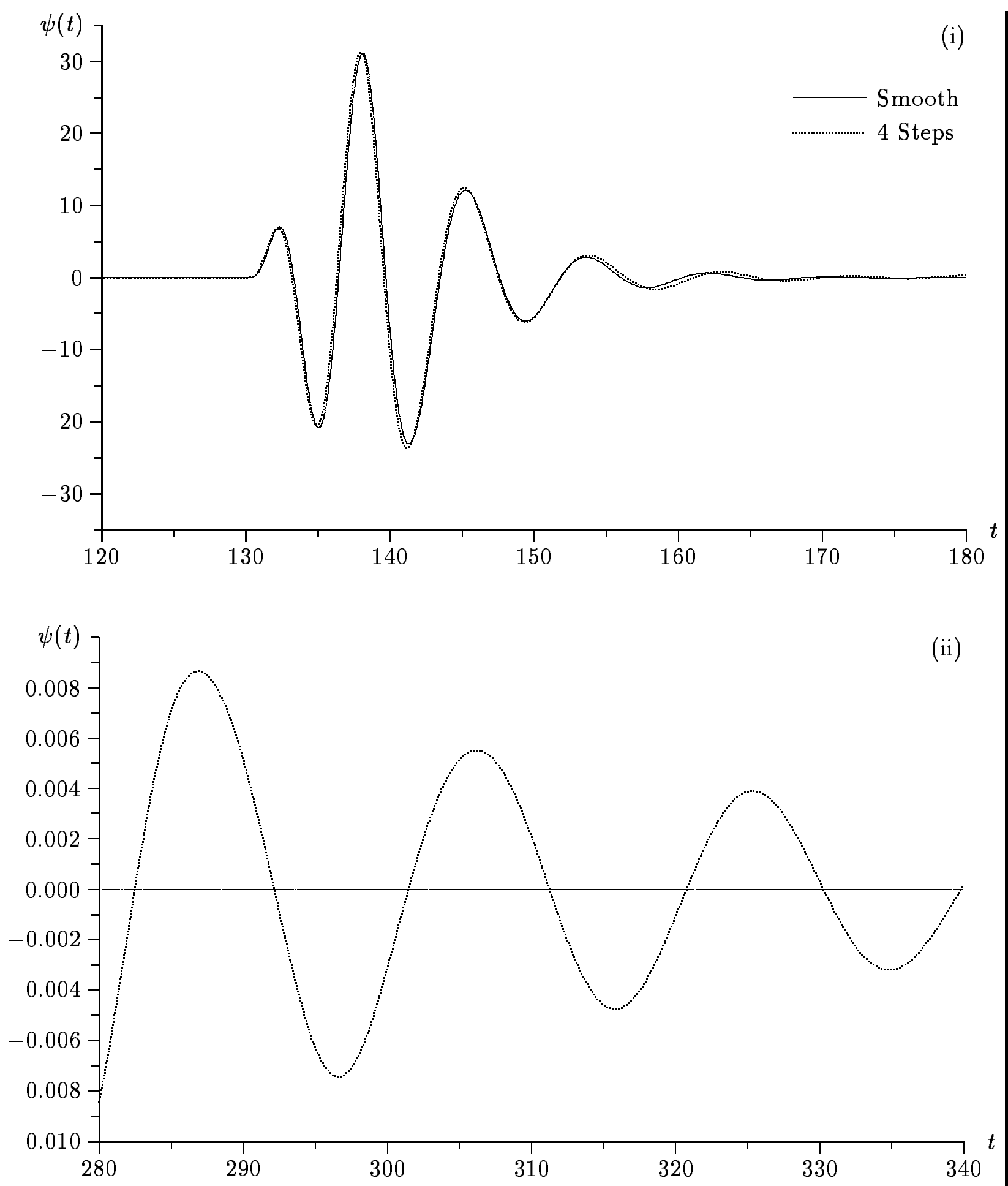

Figure 3: Time evolution of initial data, observed at $x=120$, using the smooth ReggeWheeler potential and the step potential with 4 steps and construction principle (i).

quasinormal frequencies of the smooth Regge-Wheeler potential. This is a necessary, but not sufficient, condition for the quasinormal modes forming a complete set for the solutions 
of the perturbation equation.

Time evolution computations confirm that the very late time behavior of some initial perturbation is indeed dominated by the fundamental quasinormal mode of the step potential under consideration, rather than by the fundamental mode or the tail belonging to the smooth potential. However, due to the exponential damping, this very late time behavior represents only a tiny fraction of the total response of a black hole, or some other system, to an initial perturbation. Most of the total response occurs at earlier times, where it is dominated by quasinormal ringing at the fundamental frequency of the smooth potential.

Our results suggest that it is indeed possible to obtain an expanded set of quasinormal modes which can completely represent the time evolution of perturbations of black holes. This conclusion increases the significance of quasinormal modes for the study of perturbations of black holes. On the other hand, we also lose something: None of the frequencies of the new modes is obvious in the largest part of the time evolution of the perturbation. It is generally assumed that at least the fundamental quasinormal frequencies have physical meaning in the sense that they will dominate the time evolution of a perturbation of the black hole, as it is known for the quasinormal frequencies of the continuous Regge-Wheeler potential. The quasinormal spectrum of a very similar step potential, on the other hand, might actually lead to a complete system of quasinormal modes, but there is no single mode or frequency which has an obvious relationship to the time dependence of the perturbation.

We have also seen that even a very small deviation from the original potential leads to a completely different quasinormal mode spectrum. It appears rather farfetched to assign significance to quantities which are so sensitive to small details, rather than to the overall features of the problem. On the other hand, this sensitivity might well be a consequence of some specific property of the step potentials, as it does not seem to occur for other cases that have been studied: Ferrari and Mashhoon [12], for example, have replaced the Regge-Wheeler potential by a Poeschl-Teller potential, approximating the Regge-Wheeler potential mainly around its maximum. They achieve good agreement between the fundamental quasinormal frequencies of the two potentials.

How, then, can we know if the quasinormal spectrum of any given system does indeed tell us something about the time evolution of a perturbation of this systems? What are the criteria which distinguish a "meaningful" from a "meaningless" spectrum? Are quasinormal frequencies always strongly sensitive to small changes in some parameters of the problem? And can we find another way to obtain an "expanded" set of quasinormal modes which is complete, but leaves the "original" modes more or less intact?

There is an intriguing parallel between the quasinormal spectrum we have found for step versions of the Regge-Wheeler potential and those of neutron stars: The frequencies of the so-called wave modes, which are associated mainly with the metric outside the star, rather than with the fluid inside, show a picture rather similar to what we have found here for the quasinormal frequencies of the step potentials, with the frequencies of the $w$ modes corresponding to the frequencies lined up along the real axis, and the frequencies of the $w_{\mathrm{II}}$-modes corresponding to the 'outlying' frequencies [2]. A better understanding of neutron star perturbations as well as Schrödinger or wave equations with discontinuities 
is required to decide whether this parallel is a coincidence or not. It is possible, however, that the junction conditions at the surface of the neutron star provide an analogy for some discontinuity in a potential.

The present work shows that the significance of the quasinormal mode spectrum of a black hole is not yet clearly understood. The most intriguing questions may be whether it is possible to "complete" the set of quasinormal modes in such a way that the fundamental modes still represent the major features of the time evolution, and how the excitation of a particular quasinormal mode can be measured, or even be given a unanimous meaning.

\section{Acknowledgments}

We wish to thank Richard Price for bringing the completeness problem to our attention, and for many enlightening discussions. Jorge Pullin made available a code which helped with the computation of the time evolution data.

\section{References}

[1] K. D. Kokkotas and B. F. Schutz, Mon. Not. R. Astron. Soc. 255, 119 (1992)

[2] M. Leins, H.-P. Nollert, and M. H. Soffel, Phys. Rev. D 48, 3467 (1993)

[3] P. T. Leung, S. Y. Liu, S. S. Tong, and K. Young, Phys. Rev. A 49, 3068 (1994)

[4] P. T. Leung, S. Y. Liu, and K. Young, Phys. Rev. A 49, 3057 (1994)

[5] W. H. Press, Ap. J. 170, L105 (1971)

[6] C. V. Vishveshwara, Nature 227, 936 (1970)

[7] S. Chandrasekhar and S. Detweiler, Proc. R. Soc. London A 344, 441 (1975)

[8] A. Bachelot and A. Motet-Bachelot, Proceedings of the "IV International Conference on Hyperbolic Problems", Taosmina, ed. Vieweg (1992)

[9] E. Leaver, Phys. Rev. D 34, 384 (1986), Phys. Rev. D 38, 725 (erratum)

[10] R. H. Price and V. Husain, Phys. Rev. Lett. 68, 1973 (1992)

[11] E. S. C. Ching, P. T. Leung, W. M. Suen, and K. Young, Phys. Rev. Lett. 74, 4588 (1995), gr-qc/9408043

[12] V. Ferrari and B. Mashhoon, Phys. Rev. Lett. 52, 1361 (1984)

V. Ferrari and B. Mashhoon, Phys. Rev. D 30, 295 (1984) 